\title{
Effects of bulking agents, load size or starter cultures in kitchen-waste composting
}

\begin{abstract}
To prevent the interruption of the carbon cycle by the disposal of waste to landfills, organic kitchen waste requires proper treatment such as composting to reduce its uncontrolled degradation on disposal sites and subsequent greenhouse gases, odour emissions and nutrient losses. This study investigated the effects of bulking agent, newspaper and onion peels, composting waste load sizes of 2 and $6 \mathrm{~kg}$, or the use of starter culture on kitchen-waste composting consisting of nitrogen-riched substrates, vegetable scraps and fish processing waste in an in-vessel system. The optimised formulation of kitchen waste mixture was used for a 30-day composting study, where the temperature profiles were recorded and the carbonto-nitrogen ratios were measured as an indication of compost maturity. The kitchen-waste composting process was conducted in parallel in two fabricated kitchen waste composters.
\end{abstract}

Keyword: Newspaper; Onion peels; Kitchen waste; Composting; Starter culture 